\title{
Pure laparoscopic hepatectomy and robotic graft implantation in living donor liver transplantation: a case report
}

Kyung-Suk Suh, Suk Kyun Hong, Sola Lee, Su young Hong, Sanggyun Suh, Eui Soo Han, Seong-Mi Yang, YoungRok Choi, Nam-Joon Yi, Kwang-Woong Lee

Department of Surgery-Transplantation, Seoul National University Hospital, Seoul, Korea

Background: Successful experiences with pure laparoscopic explant hepatectomy and graft implantation using upper midline incision have allowed us to extend our minimally invasive living donor liver transplantation (LDLT) program to pure laparoscopic explant hepatectomy and pure laparoscopic and/or robotic graft implantation using suprapubic incision. Here, we share our initial experience of using pure laparoscopic explant hepatectomy followed by pure laparoscopic and/or robotic graft implantation. Case report: A 51-year-old male required a liver transplant for autoimmune hepatitis-related liver cirrhosis. Pure laparoscopic explant hepatectomy followed by pure laparoscopic and/or robotic graft implantation was performed. The time to remove the native liver was 260 minutes, and the total operative time was 1,065 minutes. The time required for anastomosis of the hepatic vein, right inferior hepatic vein, portal vein, hepatic artery, and bile duct were $41,26,37,123$, and 72 minutes, respectively. Protocol computed tomography performed on postoperative day 7 revealed no abnormal findings. The patient was discharged on postoperative day 13 , with no complications.

Conclusions: Pure laparoscopic explant hepatectomy followed by pure laparoscopic and robotic graft implantation in LDLT can be performed. 